Proc. 15th Int. Conference on Defects Recognition, Imaging and Physics in Semiconductors, Warsaw, Poland 2013

\title{
Residual Strain and Electrical Activity of Defects in Multicrystalline Silicon Solar Cells
}

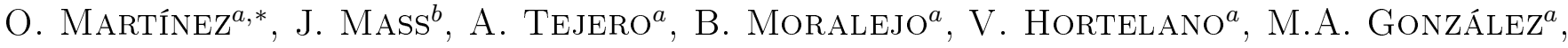 \\ J. JiMÉNEZ ${ }^{a}$ AND V. PARRA ${ }^{c}$ \\ ${ }^{a}$ GdS-Optronlab, Dpto. Física Materia Condensada, Parque Científico Univ. de Valladolid, 47011 Valladolid, Spain \\ ${ }^{b}$ Departamento de Física, Grupo de Física Aplicada, Universidad del Norte \\ Km. 5-Vía Puerto Colombia, Barranquilla, Colombia \\ ${ }^{c}$ DC-Wafers Investments, S.L. Ctra. de Madrid, Km. 320, 24227 Valdelafuente, León, Spain
}

\begin{abstract}
The growth process by casting methods of multi-crystalline Si results in a crystalline material with, among other defects, a high density of dislocations and grain boundaries. Impurity incorporation and their gathering around grain boundaries and dislocations seem to be the main factor determining the electrical activity of those defects, which limit the minority carrier lifetime. In this work, we analyze multi-crystalline Si samples by combining etching processes to reveal the defects, Raman spectroscopy for strain measurements, and light beam induced current measurements for the localization of electrically active defects. In particular, we have explored the etching routes capable to reveal the main defects (grain boundaries and dislocation lines), while their electrical activity is studied by the light beam induced current technique. We further analyze the strain levels around these defects by Raman micro-spectroscopy, aiming to obtain a more general picture of the correlation between residual stress and electrical activity of the extended defects. The higher stress levels are observed around intra-grain defects associated with dislocation lines, rather than around the grain boundaries. On the other hand, the intra-grain defects are also observed to give dark light beam induced current contrast associated with a higher electrical activity of these defects as compared to the grain boundaries.
\end{abstract}

DOI: $10.12693 /$ APhysPolA.125.1013

PACS: 78.30.Am, 61.72.Ff, 88.40.jj

\section{Introduction}

Multi-crystalline $\mathrm{Si}$ (mc-Si) grown by casting methods, despite the renewed interest in the Czochralski Si based materials to develop high efficiency solar cells, is still the preferred material for terrestrial photovoltaic (PV) applications due to its cost-effectiveness. Mc-Si combines a convenient product cost and high final efficiencies [1]. However, it is well known that the growth process by casting methods results in a crystalline material with a high density of dislocations generated by the mechanical mismatches during the cooling down process, as well as a large number of grain boundaries (GBs). Impurity incorporation and their gathering around GBs and dislocations seem to be the main problem limiting the minority carrier lifetime $[2,3]$. The consequence is a material with lower efficiency as compared to mono-crystalline silicon, with the concomitant loss of efficiency with respect to the most pure material (17-18\% vs. 19-20\%) [1]. During the last years, an important effort is being made in the improvement of mc-Si wafers, regarding both the diminution and passivation of extended and punctual defects [4]. The implementation of experimental tools allowing for the localization of such defects, exploring their local distribution and origin, is a step forward to the understand-

*corresponding author; e-mail: oscar@fmc.uva.es ing of the role of the crystal defects in the performance of the mc-Si based solar cells.

In this work, we analyze mc-Si samples by combining etching processes to reveal the defects, Raman spectroscopy for strain measurements, and light and beam induced current (LBIC-EBIC) measurements for the localization of electrically active defects. Raman spectroscopy is a well-known characterization tool providing valuable information about crystalline quality of semiconducting materials. However, it has not been until recently that it is gaining importance for the characterization of solar Si due to its capability to map the local residual stresses [5-7], which are critical to the mechanical stability, especially in view of silicon wafer thickness reduction, but also might play a crucial role in the distribution of the metallic impurities, responsible for the minority carrier lifetime killing.

The direct observation of the crystal defects (GBs and intra-grain defects) at the same time that the measurement of their electrical activity and associated residual stress is not an easy task. The electrical characterization can be obtained by LBIC measurements on the final cell (Fig. 1), but no information about the specific origin of the observed defects is obtained. Moreover, the Raman spectrum of final solar cells is contributed by the presence of the additional layers and texturizing treatment of the surface, borrowing the information about the bare mc-Si. For these reasons, we have developed a methodological analysis, on bare mc-Si wafers, in order to directly visu- 
alize the crystal defects and at the same time be able to obtain information about their electrical activity and/or the associated residual stress. The surface of sliced mc-Si wafers does not permit to extract clean Raman information; therefore, surface preparation is necessary in order to obtain the Raman spectra free of the influence of the surface.

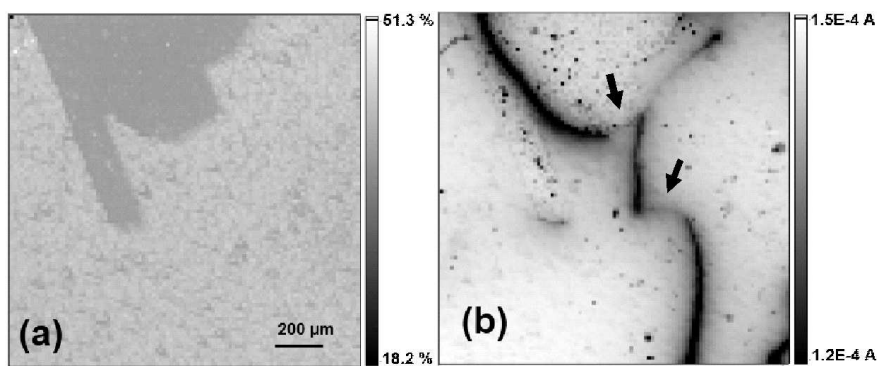

Fig. 1. Reflectivity image (a) and LBIC map (b) of a portion of a mc-Si solar cell (objective $20 \times, \lambda_{\text {exc }}=$ $830 \mathrm{~nm})$.

In a previous work [8], we investigated different chemical etchants of polished mc-Si wafer, $\mathrm{KOH}$ etching was seen to be quite sensitive to reveal the GBs. After this GB revelation, the samples were metallised with semi-transparent gold layers on both faces for LBIC measurements; the GBs were shown to give very low LBIC contrast, concluding that the GBs themselves are not the main electrically active defects in $\mathrm{mc}-\mathrm{Si}$; on the other hand, a large number of intra-grain defects were revealed in the LBIC maps, accounting for its large electrical activity [8]. Following this investigation, we performed complementary surface etchings in order to reveal both the GBs and dislocation lines (DLs), as well as to obtain information on their associated residual stress fields and/or electrical activity, in order to establish a relation between stress and electrical activity.

\section{Experimental and samples}

Both mc-Si wafers, grown by conventional casting, and solar cells fabricated following standard industrial methods, have been investigated. Casting mc-Si growth was performed at DC Wafers Investments, S.L., Spain, using both directional solidification (DSS) and heat exchange method (HEM) furnace stations (from GT Solar, USA). P-type mc-Si bricks (B-doped) with resistivities in the range of $1-2 \Omega \mathrm{cm}$ were grown, conventional square shaped wafers $\left(156 \times 156 \mathrm{~mm}^{2}\right)$ were cut from these bricks. Solar cells were manufactured using $\mathrm{HNO}_{3} /$ HF-based texturing processes and a typical H-patterned screen-printing metallization. $P$-diffusion was performed by both inline $\left(\mathrm{H}_{3} \mathrm{PO}_{4}\right)$ and offline $\left(\mathrm{POCl}_{3}\right)$ methods.

In order to reveal the main crystal defects (GBs, DLs, etc.), we carried out a combination of chemical etchings using $\mathrm{HF}: \mathrm{HNO}_{3}: \mathrm{CH}_{3} \mathrm{COOH}$ (chemical polishing), $\mathrm{KOH}$ (alkaline etching), and Secco attacks on polished mc-Si wafers. Different combinations and times have been probed. LBIC measurements with a home-made system were performed on the as received final solar cells as well on the etched mc-Si wafers, after a gold metallization on both sides, as described in [8]. The LBIC system operates with four excitation wavelengths (639-830 $\mathrm{nm}$ and $853-975 \mathrm{~nm}$ dual emission lines). Excitation is typically made with low laser powers, ensuring that the majority carrier density is not significantly affected. The reflected optical image (visualized by a Si-CCD camera) and the photocurrent signal are monitored simultaneously. A motorized $x-y$ stage allows for mapping the LBIC signals; the use of different microscope objectives allow for mapping large areas of the wafers $(20 \times)$, and to perform high spatial resolution LBIC maps $(100 \times)[9,10]$. Both room temperature (RT) and low temperature measurements were carried out. EBIC measurements at RT were also carried out on a field emission scanning electron microscope (FESEM) (Carl Zeiss-LEO 1530) using typical beam energies of $30 \mathrm{keV}$. The electrical current generated by the electron beam is collected and amplified by a low noise current amplifier (MODEL DLPCA-200, FEMTO Messtechnik, Germany).

The Raman spectra were acquired at room temperature using the $532 \mathrm{~nm}$ line of a frequency doubled $\mathrm{Nd}$ -YAG laser, exciting the sample through a $50 \times$ long working distance microscope objective, in backscattered configuration. The scattered light was analysed by means of a Raman spectrometer (Labram HR800 UV from Horiba-Jobin-Yvon) equipped with a $\mathrm{LN}_{2}$-cooled charge-coupled device detector. The measurements were done at RT. A motorized $x-y$ stage allows for mapping the Raman signal. The spectra were fitted by Lorentzian curves, obtaining maps of the Raman parameters (intensity, peak position and full width at half maximum FWHM). Because of the small Raman peak shifts to be measured, the use of a careful spectral reference setup is mandatory in order to correct thermal and mechanical instabilities of the system along the measurement. For this purpose, a neon lamp located inside the optical entrance of the spectrometer, just ahead of the entrance slit, was used as an internal reference; this procedure warrants a very stable spectral reference insensitive to the beam alignment.

\section{Results}

Typical LBIC measurements performed on conventional final mc-Si solar cells generally showed that the GBs are not the main electrically active defects, while a large number of intra-grain defects, some of them with a very large electrical activity, are usually observed, see Fig. 1. In the optical images acquired simultaneously to the LBIC signal, the GBs are revealed through the different reflectivity observed for each of the different grains (see the reflectivity map in Fig. 1a); also, for medium and high spatial resolution LBIC maps, the texture of the different grains is well observed. 
The intra-grain defects are only observed here through their electrical activity. They are supposed to be DLs, around which impurities can be captured by their strain fields, with the subsequent recombination of the photogenerated carriers, giving the dark contrast in the LBIC maps. It is interesting to note that the electrical activity of the intra-grain dark lines is not homogeneous, but seems to depend on the line orientation, see the segments marked by arrows, Fig. 1b. This is a quite general observation in the LBIC images recorded in mc-Si solar cells: Fig. 2 shows high resolution EBIC maps obtained on a solar cell, showing again the presence of intra-grain defects (not correlated to GBs, see Fig. 2a, b) with different electrical activities depending on the angle of the defective segment (Fig. 2d). This effect is likely due to the different incorporation of impurities depending on the boundary orientation of the intra-grains.
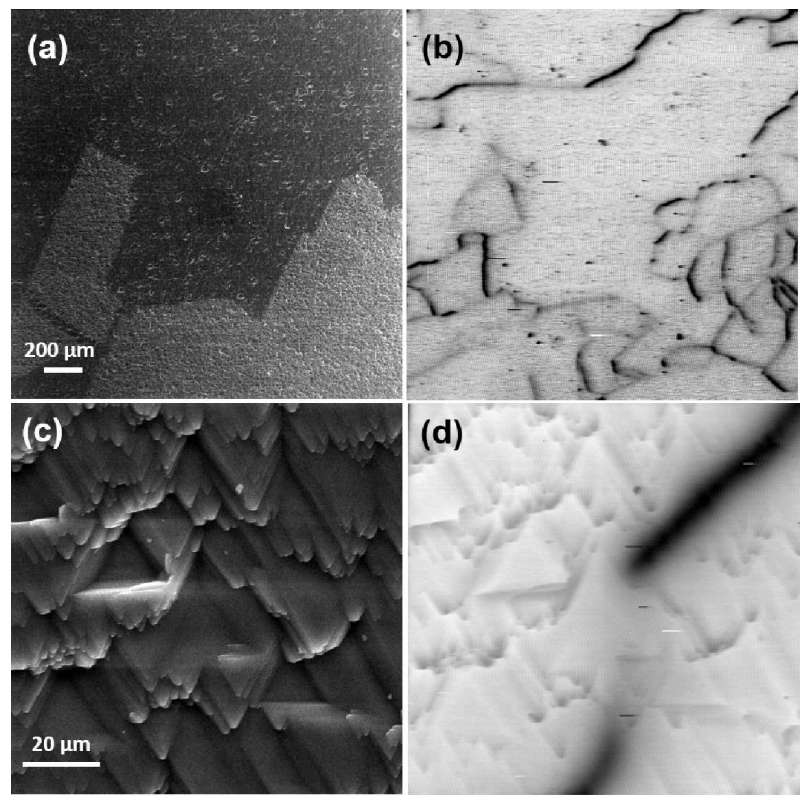

Fig. 2. SEM (a, c) and EBIC (b, d) maps of a portion of a mc-Si solar cell $(a, b)$ and of a small portion of the same cell $(\mathrm{c}, \mathrm{d})\left(E_{\mathrm{b}}=30 \mathrm{keV}, \mathrm{RT}\right)$.

In order to obtain a more direct correlation between the defects, its electrical activity and their residual stress fields, we checked the capabilities of different etchants to reveal the defects and to prepare the samples in such a way that the LBIC and Raman maps would be consecutively obtained. Among the different chemical solutions and combinations probed, we observed that the combination of a short chemical polishing, followed by a medium Secco attack and a final short alkaline etching is the best one to reveal the different defects, see Fig. 3.

The combination of a Secco attack and an alkaline etching seems to be able to reveal both the dislocations and DLs (through the Secco attack) and the GBs and sub-GBs (by the alkaline etching). The previous chemical polishing seems to be important to prepare conveniently

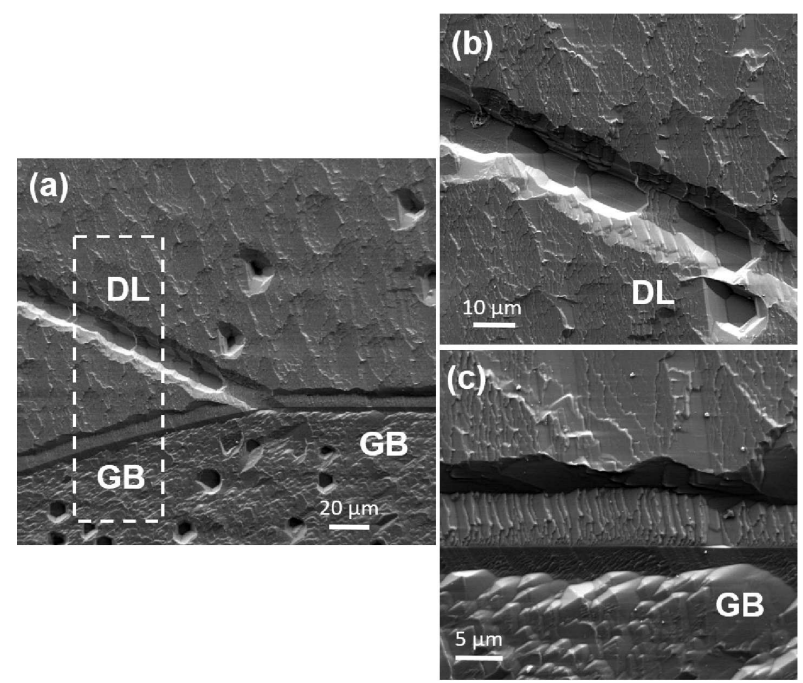

Fig. 3. SEM image of a portion of an etched mc-Si wafer (a) and enlarged SEM images arround a DL and a GB (b, c). The different textures of the grains are well observed in (c).

the surface for such etchings (Secco + alkaline). One can see the different surface textures corresponding to different grains, Fig. 3c. On the other hand, it is interesting to note the different shapes of the revealed GB and DL. The assignment of the DL has been performed since it is an intra-grain defect (the same texture is observed at both sides of the defect) and because this line appears to be a superposition of etched groves, which are the typical features revealed by this etching in dislocations [11].
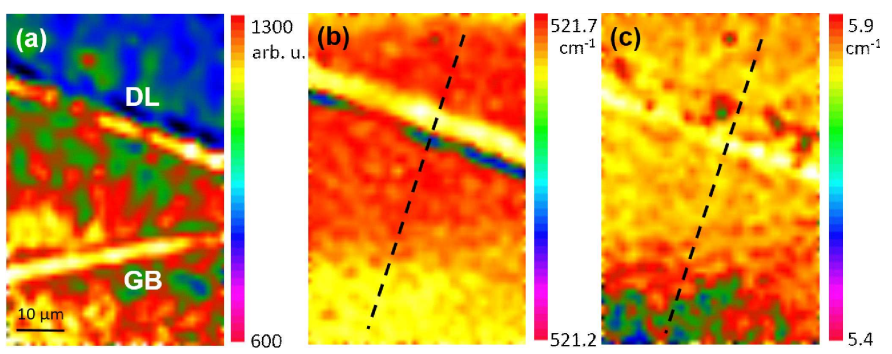

Fig. 4. Maps of the Si Raman band intensity (a), peak position (b) and FWHM (c) obtained on the square dotted region marked in Fig. 3a.

Figure 4 shows the Raman maps obtained on the rectangular region marked in Fig. 3a. Figure $4 \mathrm{a}-\mathrm{c}$ shows the results of the Lorentzian fitting (intensity, peak position and FWHM) of the Raman spectra obtained on a fine matrix of points obtained by scanning the area of interest. The intensity map reveals quite well the different textures of the two grains, since the Raman intensity is sensitive to the grain orientation. The peak position map, on the other hand, shows no contrast related to the GB line, but the contrast related to the DL is clearly observed, evidencing an accumulation of stress around 
it. The Raman profiles obtained along the dotted lines marked in Fig. 4b are shown in Fig. 5a. One can observe a slightly different peak position for each grain (521.5 and $521.6 \mathrm{~cm}^{-1}$, respectively, for the upper and lower grains), which could be correlated to a slightly different defect incorporation depending on the grain orientation. There is not a change in the stress level associated with the GB itself. Quite the opposite, important fluctuations of the residual stresses are detected around the DL. Moreover, a compressive/tensile behavior is observed around this defect. These results reveal residual strain around the DL, while the potential residual strain associated with the GB line would be below of our detection limit. One can argue that metallic impurities can decorate the DLs under the strain field created around them, as revealed by our Raman mapping measurements.

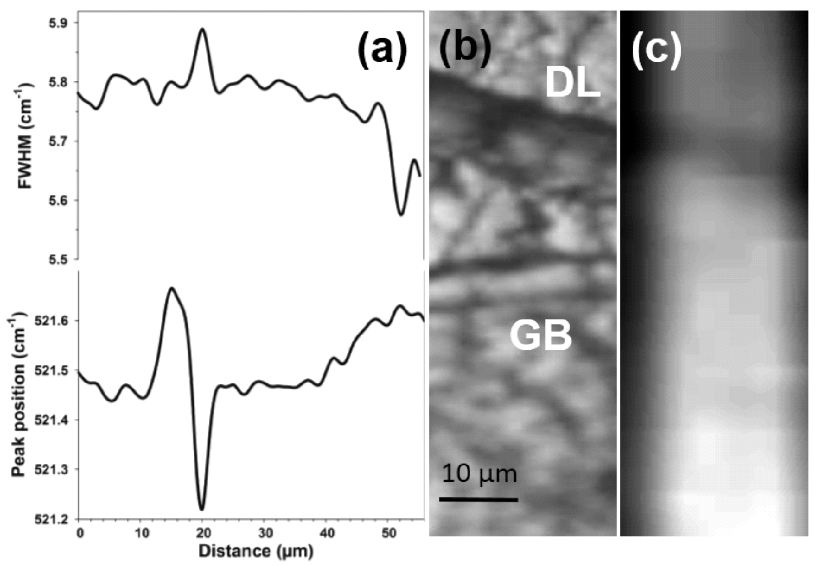

Fig. 5. Raman profiles (peak position and FWHM) along the dotted line marked in Fig. 4b,c; (b) optical image corresponding to the same area studied in Fig. 4, (c) LBIC map obtained at $80 \mathrm{~K}$ on the same area.

An estimation of the residual stress can be deduced from the Raman shift, according to the equation (for a silicon sample under biaxial stress on (100) plane [12]):

$$
\sigma(\mathrm{MPa})=-250 \Delta \omega\left(\mathrm{cm}^{-1}\right) \text {. }
$$

According to this expression, a change of $\pm 50 \mathrm{MPa}$ is detected around the DL.

The electrical behavior of the analyzed area was also measured by LBIC after gold metallization on both sides. The electrical contrast response was very low at RT, likely due to the very small thickness of the samples after polishing and etching. LBIC measurements were also carried out at low temperature $(80 \mathrm{~K})$ in order to improve the sensitivity contrast, Fig. 5 b,c. One can observe that the DL of Fig. $5 \mathrm{c}$ gives a tenuous dark contrast as compared to the absence of contrast along the GBs, indicating that the DL exhibits a higher electrical activity together with a higher strain field.

\section{Conclusions}

The electrical activity of extended defects observed in mc-Si were correlated to their strain field mapped by $\mu$ Raman measurements. The Raman maps obtained on an area showing both GBs and DLs, revealed by chemical etching, showed different strain fields for both types of defects. While the GBs show strain levels below the detection limit of our experimental setup, the DL show a marked tensile/compressive behaviour, with stress values of $\pm 50 \mathrm{MPa}$ around the DL. The electrical activity of the analysed area was also studied by LBIC measurements at low temperature, where a tenuous dark contrast was revealed around the DL, while no contrast was observed for the GB. Thus, the higher electrical activity of the intra-grain (DL) defects with respect to the GBs can be explained by the different strain fields. Metallic impurities, which are responsible for the dark LBIC contrast, would decorate the DLs under the influence of the strain field associated to them.

\section{Acknowledgments}

This work was supported by the Research Projects VA166A11-2 ("Consejería de Educación, Junta de Castilla y León" - Spain) and IPT-420000-2010-022 (INNPACTO program, "Ministerio de Ciencia e Innovación", Spain).

\section{References}

[1] 6th Report on Solar Generation: Solar Photovoltaic Electricity Empowering the World, European Photovoltaic Industry Association - EPIA Publications, 2011.

[2] N. Chen, S. Qiu, B. Liu, G. Du, G. Liu, W. Sun, Mater. Sci. Semicond. Proc. 13, 276 (2010).

[3] M. Rinio, A. Yodyungyong, S. Keipert-Colberg, D. Borchet, A. Montesdeoca-Santana, Phys. Status Solidi A 208, 760 (2011).

[4] E. Schmid, S. Würzner, C. Funke, V. Galindo, O. Pätzold, M. Stelter, J. Cryst. Growth 359, 77 (2012).

[5] T. Tachibana, J. Masuda, A. Ogura, Y. Ohshita, K. Arafune, ECS Trans. 25, 33 (2010).

[6] M. Becker, H. Scheel, S. Christiansen, H.P. Strunk, J. Appl. Phys. 101, 063531 (2007).

[7] G. Sarau, S. Christiansen, M. Holla, W. Seifert, Sol. Energy Mater. Sol. Cells 95, 2264 (2011).

[8] J. Domínguez, J. Mass, B. Moralejo, O. Martínez, J. Jiménez, A.M. Ardila, V. Parra, J. Mater. Sci. 47, 5470 (2012).

[9] J. Jiménez, B. Moralejo, V. Hortelano, M.A. González, O. Martínez, V. Parra, M. Avella, Mater. Res. Soc. Symp. Proc. 1268, EE01-08.R1 (2010).

[10] B. Moralejo, V. Hortelano, M.A. González, O. Martínez, J. Jiménez, S. Ponce-Alcántara, V. Parra, Phys. Status Solidi C 8, 1330 (2011).

[11] F. Secco d'Aragona, J. Electrochem. Soc. 119, 948 (1972).

[12] I. DeWolf, Semicond. Sci. Technol. 11, 139 (1996). 